\title{
The Development of Mauna Kea as an Astronomical Site Panelists: John Jefferies, Ann Boesgaard, Alan Stockton, Eric Becklin, and Alan Tokunaga
}

\author{
Teasel Muir Harmony ${ }^{1}$ and David DeVorkin ${ }^{2}$ (moderators) \\ ${ }^{1}$ Center for History of Physics, American Institute of Physics, College Park, MD 20740, USA \\ email: tmuir@aip.org \\ ${ }^{3}$ National Air and Space Museum, Smithsonian Institution, Washington, DC 20560, USA \\ email: devorkind@si.edu
}

\begin{abstract}
On August 11 we held a panel discussion at the 2015 IAU General Assembly, within the three-day Focus Meeting FM2, "Astronomical Heritage: Progressing the UNESCO-IAU Initiative". Our purpose was to both honor and explore the contributions of John Jefferies to the creation and development of Mauna Kea as an astronomical site.
\end{abstract}

\section{Overview}

The panel format (see Fig. 1) provided a platform wherein these astronomers, and the audience, shared their recollections and views relating to the history of the establishment of Mauna Kea and the Institute for Astronomy and Jefferies' role in bringing both into existence. Organizing the panel also provided the opportunity for identifying and assessing the state of preservation of the historical record covering the early history of modern astronomy in Hawaii.

DeVorkin opened the session with a short illustrated overview (see the pdf file on the FM2 website www.astronomicalheritage.net/index.php/community/news-events/focusmeeting-at-iau-general-assembly, which also contains Becklin's presentation) and then invited Jefferies to provide a short account of what attracted him to Hawaii. His remarks were then followed by remarks and queries by those noted in the order above, followed by responses by Jefferies. Finally Muir-Harmony posed general questions to the panel and moderated audience responses.

Principal questions posed to, and by, each of the panelists during the session, and in more depth during oral history sessions held during the meetings, are:

1. What were the chief opportunities, challenges and then hurdles to overcome in the identification and then implementation of Mauna Kea as an ideal site?

2. How did the University of Hawaii become the host institution for its establishment?

3. What was learned from tracking sites established on Haleakela by the U. S. Air Force and the Smithsonian that focused attention on Mauna Kea?

4. How and why did NASA become interested in establishing a large observatory there?

5. And once the site was established and its qualities fully appreciated, how did astronomical institutions from all over the world become attracted to the site? 


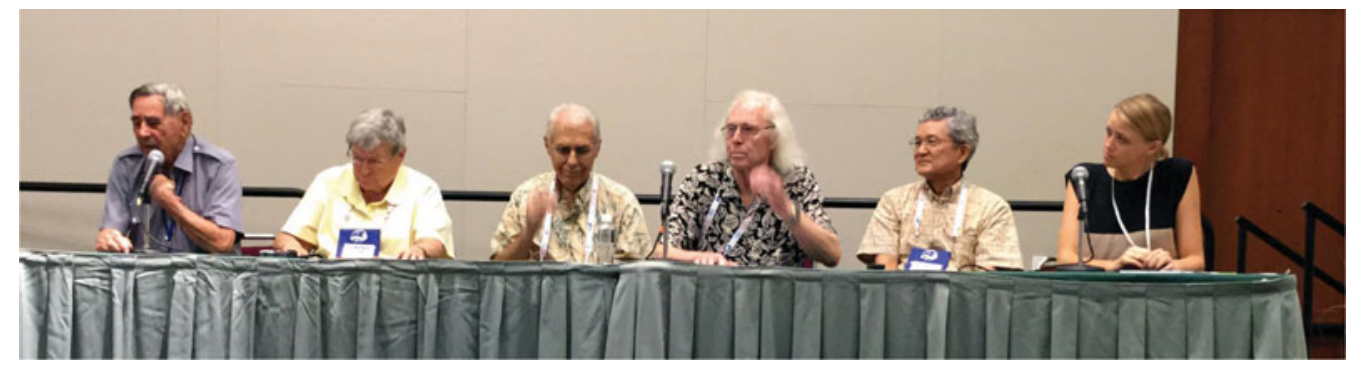

Figure 1. The panel. From left to right: John Jefferies, Ann Boesgaard, Alan Stockton, Eric Becklin, Alan Tokunaga, and Teasel Muir Harmony

The panel discussion was recorded as were all the interview sessions, which are now being processed by the American Institute of Physics. All of the panelists, as well as two other participants in the early years, were interviewed privately during the first and second weeks of the meeting. The resulting 25 hours of interviews as well as findings during an inspection tour of the archival vault maintained by the Institute for Astronomy will, we hope, stimulate increased attention by historians to the history of modern astronomy in Hawaii. 